\title{
EchoGéo
}

$53 \mid 2020$

Dénominations plurielles. Quand les noms de lieux se concurrencent

\section{Nommer les lieux de la crise des opioïdes à Boston : un enjeu politique}

Naming the sites of the opioid crisis in Boston: a political issue

\section{Elsa Vivant}

\section{OpenEdition}

\section{Journals}

Édition électronique

URL : https://journals.openedition.org/echogeo/20001

DOI : 10.4000/echogeo.20001

ISSN : 1963-1197

Éditeur

Pôle de recherche pour l'organisation et la diffusion de l'information géographique (CNRS UMR 8586)

Référence électronique

Elsa Vivant, « Nommer les lieux de la crise des opioïdes à Boston : un enjeu politique », EchoGéo [En ligne], 53 | 2020, mis en ligne le 30 septembre 2020, consulté le 11 août 2021. URL : http:// journals.openedition.org/echogeo/20001; DOI : https://doi.org/10.4000/echogeo.20001

Ce document a été généré automatiquement le 11 août 2021.

EchoGéo est mis à disposition selon les termes de la licence Creative Commons Attribution - Pas d'Utilisation Commerciale - Pas de Modification 4.0 International (CC BY-NC-ND) 


\title{
Nommer les lieux de la crise des opioïdes à Boston : un enjeu politique
}

Naming the sites of the opioid crisis in Boston: a political issue

\author{
Elsa Vivant
}

In memorium Aubri Ester

\section{Nommer les lieux de la crise des opioïdes à Boston : un enjeu politique}

Le 14 octobre 2014, le Maire de Boston a pris la décision de fermer à toute circulation le pont qui menait à l'ile de Long Island impliquant la fermeture et le déménagement, dans la journée, de l'ensemble des services aux plus démunis localisés de longue date sur cette ile. En quelques heures, le foyer d'hébergement d'urgence pour sans-abris et les centres de soins en addictologie ont dû relocaliser leurs activités, dans des conditions le plus souvent précaires, ailleurs dans l'agglomération. Non préparé, ce déménagement en urgence a contribué à la fragilisation des conditions de vie des sansabris, beaucoup ne trouvant pas de solution d'hébergement, et à la constitution d'une scène ouverte de consommation de drogues localisée dans un secteur au croisement de deux grandes artères (Massachusetts Avenue et Melnea Cass Boulevard) à l'interface de trois quartiers aux usages et dynamiques contrastées : une zone de petites industries et de stockage (NewMarket), un quartier populaire noir et latino (Dudley-Roxbury) et un quartier gentrifié (South End). La décision du maire est intervenue au moment où la crise des opioïdes est devenue un problème public, politique et médiatique ${ }^{1}$. Latente depuis la fin des années 1990, l'introduction sur le marché clandestin d'un opioïde de synthèse puissant (le Fentanyl) a contribué à l'aggravation de la crise par une croissance dramatique de la mortalité par surdose ${ }^{2}$. 
2 La scène ouverte focalise l'attention des médias et des pouvoirs publics en rendant visible les problèmes d'addiction et d'overdose et en raison des conflits d'usage de l'espace public qu'elle génère. Elle est l'objet de différentes nominations, renvoyant chacune à des représentations différentes du problème et porteuses d'enjeux géopolitiques locaux. Entrer sur cette scène par ses noms s'avère être particulièrement heuristique pour dénouer la complexité de cette crise sanitaire, de ses enjeux politiques et des infléchissements qu'elle provoque dans la politique des drogues. Comme le rappellent plusieurs auteurs (Giraut et Houssay-Holzschuch, 2008; Rose-Redwood et Alderman 2011), l'acte de nommer un lieu est politique et les débats relatifs à la toponymie constituent un analyseur des dynamiques sociales, politiques (et ici sanitaires) qui se jouent sur un espace. Dans cet article, la pluralité des nominations est un instrument heuristique dans la compréhension de la crise des opioïdes et des effets sur les représentations sociales des usagers de drogues. Les débats sur les manières de nommer (les lieux mais aussi les traitements ou les usagers de drogues) mettent au jour l'émergence de nouvelles pratiques de soin et représentations politiques de la drogue et de ses usagers.

3 Cet article s'appuie sur une enquête approfondie, menée entre septembre 2018 et juin 2019, qui a donné lieu à la réalisation de 70 entretiens $^{3}$ et à l'observation d'autant de réunions de différentes natures ${ }^{4}$. L'article s'organise autour des trois toponymes les plus utilisés mais, parfois, d'autres nominations entreront en jeu, à différentes échelles (du bâtiment au quartier). Derrière son apparente neutralité (Rose-Redwood et Alderman, 2011), la référence à son adressage, Mass \& Cass, renvoie à l'histoire urbaine, coloniale et raciale de la ville et de la prohibition. Deux visions de l'addiction et du soin se disputent derrière d'autres noms. La localisation de nombreux services de soin ou d'encadrement des consommateurs de drogues (prison du comté et ses services de probation, cliniques de méthadone, foyer d'hébergement, centre d'hébergement d'urgence, accueil de jour) ont conduit les critiques à surnommer le secteur Methadone Mile. Les soignants, eux, préfèrent l'appeler Recovery Road, en raison de l'espoir que la présence de ces services rendrait possible.

\section{Mass \& Cass : derrière la toponymie officielle, l'impensé colonial}

4 Pour désigner la scène ouverte, l'usage officiel est de faire référence à sa localisation géographique par son adressage au croisement de deux grandes artères routières: Massachusetts Avenue et Melnea Cass Boulevard, souvent raccourci en Mass \& Cass. Cette toponymie officielle renvoie aux pages les plus sombres de l'histoire américaine : la colonisation et la ségrégation raciale. Massachusetts Avenue traverse l'agglomération, d'Arlington à Dorchester, longeant les campus du MIT et de Harvard (à Cambridge), suivant l'axe de la gentrification de South Bay au South End, s'enfonçant ensuite dans les quartiers industriels de New Market. Massachusetts est surtout le nom du peuple autochtone qui vivait sur ces terres avant l'arrivée des Européens, décimé dès les premières années de la colonisation, dont seuls quelques dizaines de descendants survivent et se battent pour faire valoir leurs droits. Melnea Cass Boulevard a été construit en 1981 sur l'emprise réservée pour la création d'une autoroute contestée par les riverains et abandonnée en 1972 (Miller, 2018). A défaut d'une autoroute, le boulevard, en deux fois trois voies, entrecoupé de feux de 
signalisation, constitue une coupure urbaine entre le centre-ville et Roxbury. Il doit son nom à Melnea Cass (1896-1978), activiste Africaine-Américaine militante pour les droits politiques, sociaux et économiques des femmes de sa communauté dans le quartier de Dudley-Roxbury, très active dans les luttes pour la déségrégation scolaire (ou busing) qui ont, à Boston, donné lieu à de violentes contestations (King, 1981; O'Connor, 2001). Preuve des enjeux politiques de la toponymie, Dudley Square, du nom d'un des fondateurs de la colonie de la Baie du Massachusetts et ancien gouverneur, accusé d'avoir possédé des esclaves noirs, a été rebaptisée Nubian Square, en 2019, en signe de reconnaissance du rôle de la population africaine-américaine dans le quartier (MacQuarrie, 2019; Greenberg, 2019). Mais cette décision intervient alors que la tendance à la gentrification du quartier a déjà commencé, au risque de mémorialiser la présence d'une population en voie d'expulsion ${ }^{5}$.

Cette référence à l'adresse est discutée par des riverains du quartier noir de DudleyRoxbury selon lesquels elle produirait une nouvelle spatialité, inventant un territoire imaginé qui quelques années plus tôt n'existait pas, occultant l'impact réel de la crise sur leur quartier et détournant les regards et l'attention de la municipalité. En réunion publique comme au cours d'entretiens, les habitants de Roxbury font part de leur ressentiment face à un problème de drogue qui, une fois de plus, concerne leur quartier, sans que, selon eux, les autorités n'en prennent la mesure. Leur colère s'exprime principalement contre la présence de seringues usagées dans un parc public (Clifford Park) et la cour d'une école (Orchard Garden School), elle-même construite dans le cadre de la rénovation du quartier Orchard Park qui était, dans les années 1980s, une des principales scènes de drogue de la ville. En 2017, les enseignants et parents d'élèves se sont constitués ainsi en groupe de pression, se réunissant deux fois par mois pour définir leur stratégie et revendications, qu'ils médiatisent sur les réseaux sociaux. Ils s'inquiètent des effets sur la santé mentale de leur enfant de la vision quotidienne de personnes s'injectant de la drogue ou ayant un comportement jugé déviant. Ils ont organisé des manifestations avec les écoliers pour réclamer l'installation de barrières autour de la cour, l'augmentation de la présence policière et le nettoyage quotidien des abords. Ils ont trouvé en Domingos DaRosa un porte-parole virulent. Entraineur d'une équipe de football, il a présenté deux fois sa candidature aux élections municipales pour porter sur l'agenda politique et médiatique les problèmes liés à l'usage de drogues dans l'espace public et aux dangers que représentent les seringues usagées. A plusieurs reprises, il a déversé au cours de réunions publiques une boite de seringues qu'il a collectées à Clifford Park où s'entraine son équipe. D'autres riverains signalent les seringues aux services de la ville par une plateforme en ligne, les photographient, les publient sur les réseaux sociaux, les comptent, les cartographient (Bearnot et al., 2018). 
Illustration 1 - Affiche invitant à une opération de ramassage de seringues et nettoyage de l'espace public

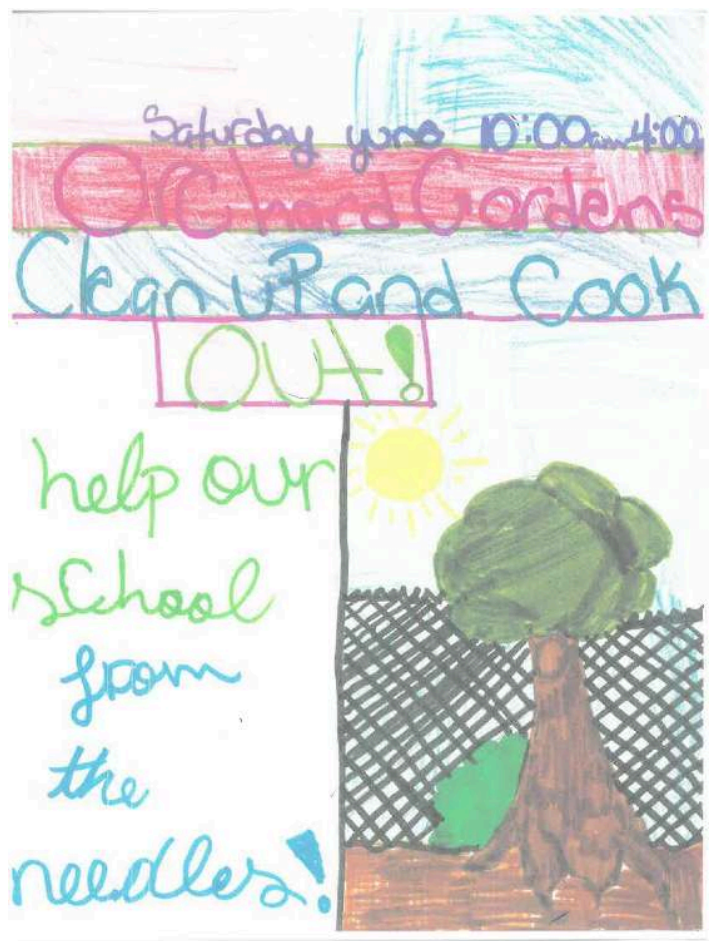

Source : réalisation par un élève anonyme de Orchard Garden School, printemps 2019.

Les réunions publiques sont l'occasion de mettre au jour les tensions et ressentiments entre les quartiers de Roxbury et du South End, mais aussi de faire prendre conscience, aux uns et aux autres, d'alliances possibles pour faire valoir le point de vue des riverains auprès de la municipalité. Pour le président d'une association de quartier du South End, malgré les différences sociales et raciales, et selon lui faisant fi des rapports de domination, gentrificateurs blancs et résidents noirs doivent s'allier pour faire la preuve de l'ampleur du problème face à la municipalité. Au contraire, les résidents de Roxbury expriment, à plusieurs reprises, leur sentiment de ne pas être écouté en raison de leur position dominée en tant que pauvres, immigrés et racisés. La possibilité d'une alliance avec les résidents du South End nierait selon eux la réalité des disparités économiques et politiques. Ils refusent l'assignation de leur quartier à être, à nouveau, la zone où les usages de drogues seraient banalisés. Leurs récriminations font également référence à un sentiment d'injustice relatif aux différences de traitement qui seraient réservées à la crise actuelle en regard des formes et effets de la guerre à la drogue qui a ciblé leur quartier par le passé. Leur sentiment est corroboré par l'analyse de Netherland et Hansen (2016) qui ont montré comment le traitement médiatique de la crise actuelle produit une représentation des usagers blancs d'opioïdes relativement bienveillante, contrastant avec la stigmatisation des usagers noirs de crack (Alexander, 2007). 


\section{Methadone Mile : toponymie officieuse de la stigmatisation}

7 À la fermeture du pont de Long Island, c'est dans ce secteur qu'un bâtiment a été rapidement transformé en foyer d'hébergement pour hommes, qu'un autre a doublé sa capacité d'accueil et que, concomitamment mais indépendamment, une seconde clinique de méthadone privée s'est installée. Ils viennent s'ajouter à d'autres services destinés ou fréquentés par les populations marginalisées et les usagers de drogues: l'hôpital et son service d'addictologie, le service d'échange de seringues, le centre municipal de santé pour sans-abris, deux cliniques de désintoxication, une clinique de méthadone privée, les services de probation et la prison du comté. Cette concentration des services contribue à la visibilité de la crise, ou plutôt à la focalisation sur son aspect le plus visible, celui ayant cours dans l'espace public, au risque d'occulter d'autres formes d'addiction. Elle est identifiée par les riverains comme la cause de l'augmentation des désordres et conflits d'usage de l'espace public. Les cliniques de méthadone sont les principales cibles des reproches et mécontentements, d'où le surnom péjoratif Methadone Mile donné au secteur, de loin la nomination la plus courante dans la presse, les conversations ordinaires ou les débats publics ${ }^{6}$.

Pour comprendre en quoi cette nomination est stigmatisante, il convient d'expliquer la suspicion que suscitent les traitements à la méthadone, expérimentés dès la fin des années 1960 à New York. Leur déploiement sous l'administration Nixon est concomitant à la criminalisation des usagers et des premières guerres à la drogue. Il s'agissait à la fois de réduire la criminalité urbaine liée à la consommation de drogue, d'anticiper les besoins de prise en charge des vétérans de retour de la guerre du Viet Nam et de répondre à la croissance de la consommation d'héroïne chez les jeunes des classes moyennes suburbaines (c'est-à-dire blanches) (White, 1998 ; Musto, 1999 ; Schneider, 2011).

9 Malgré son efficacité défendue par le corps médical, le principe même de traitements médicamenteux de l'addiction reste controversé aux Etats-Unis où le paradigme de l'abstinence et le modèle des narcotiques anonymes dominent l'offre de soin. Selon ces critiques, la méthadone ne serait qu'une drogue comme une autre, enfermant les individus dans une dépendance chronique, sans agir sur les causes psychologiques et le contexte social de la consommation. Un autre registre de critique concerne le manque de professionnalisme et la cupidité des cliniques de méthadone dans un marché peu régulé où l'un accès au soin reste discriminé (White 1998). La médiatisation des pratiques abusives de certaines cliniques (manque de suivi des patients, de contrôle des substances prescrites, de surfacturation) contribue à la mauvaise image de ce mode de traitement (Platt et al., 1998). Le protocole de soin oblige les usagers à se rendre quotidiennement dans une clinique spécialisée pour prendre leur dose devant un professionnel de santé. C'est seulement dans un second temps qu'ils peuvent emporter quelques jours de traitement à leur domicile. Ce protocole implique que les cliniques sont quotidiennement fréquentées par leurs patients, ici évalués par les riverains à plus de 1300. Ces décomptes (non sourcés) sont au centre de leur argumentation et alimentent leurs inquiétudes vis-à-vis de l'installation d'autres dispositifs de soin. La critique des traitements à la méthadone et des cliniques dédiées, souvent localisées dans des zones de relégation en raison des contraintes imposées par les règlements sanitaires ou d'urbanisme, reformule ainsi l'incarnation du déclin urbain par l'héroïne, 
les cliniques étant accusée d'attirer les dealers profitant d'une clientèle captive (Schneider, 2011).

Si la proximité de l'hôpital et de services sociaux a toujours attiré des populations marginalisées dans le South End, les riverains observent depuis 2014 un changement dans leur nombre et leur comportement dans l'espace public qu'ils attribuent à l'accentuation de l'effet d'agglomération suite à la relocalisation des services de Long Island. En bons voisins (Tissot, 2011), ils s'informent et cherchent des solutions compréhensives au problème d'usage de drogue dans l'espace public. Les représentants des associations de quartier ont participé à des réunions de travail avec les autorités de la ville et la direction de l'hôpital, pour comprendre le problème, ses causes, et identifier des solutions. Pour résorber les conflits d'usage de l'espace public et retrouver la qualité de leur cadre de vie, ils en viennent à proposer une meilleure distribution géographique des structures de soin et un assouplissement des modalités de traitement. Ils rejoignent en cela les appels des élus de Boston aux autres collectivités pour prendre en charge ce qu'ils considèrent être leur part de la charge du problème ; l'ampleur de la crise dans leur ville s'expliquant, selon eux, par l'absence de services de soin dans les autres villes et l'abandon par les autres communautés de leurs toxicomanes.

D'autres dénominations attirent l'attention, comme l'expression Ground Zero (en référence au site des attentats du 11 septembre 2001) utilisée par New Market Association pour souligner l'importance de la crise à laquelle le quartier fait face. Cette association d'entreprises formule une autre proposition: la création d'un Business Improvement District qui privatiserait la gestion de l'espace public et ses usages pour prendre en charge un problème que la ville ne semble pas pouvoir résoudre seule. Pour cela, il faut obtenir l'autorisation de la Ville et convaincre une majorité d'entreprises à payer une surtaxe foncière dédiée à la gestion du BID qui assurerait des services de sécurité et de nettoyage privés. Concentrant son intervention sur le périmètre de la zone industrielle et de ses immédiats abords, les quartiers résidentiels de Roxbury et du South End en seraient exclus ${ }^{7}$. Un tel périmètre de gestion privatisée de l'espace public viendrait par ailleurs s'ajouter à ceux existants aux alentours des propriétés de l'université et de l'hôpital où interviennent, en plus de la police municipale et d'Etat, des polices privées qui, selon des militants de la cause des sans-abris, exerceraient une violence institutionnelle par des pratiques discriminatoires de harcèlement (délogement des sans-abris, restriction d'accès...). Ces différents périmètres de gestion de l'espace public viennent renforcer les effets de frontières produits par les coupures urbaines et les différentes délimitations de circonscriptions administratives, électorales, politiques et policières qui ne se superposent pas. 
Illustration 2 - La scène ouverte : une concentration de services destinés aux usages de drogues aux confins de trois quartiers

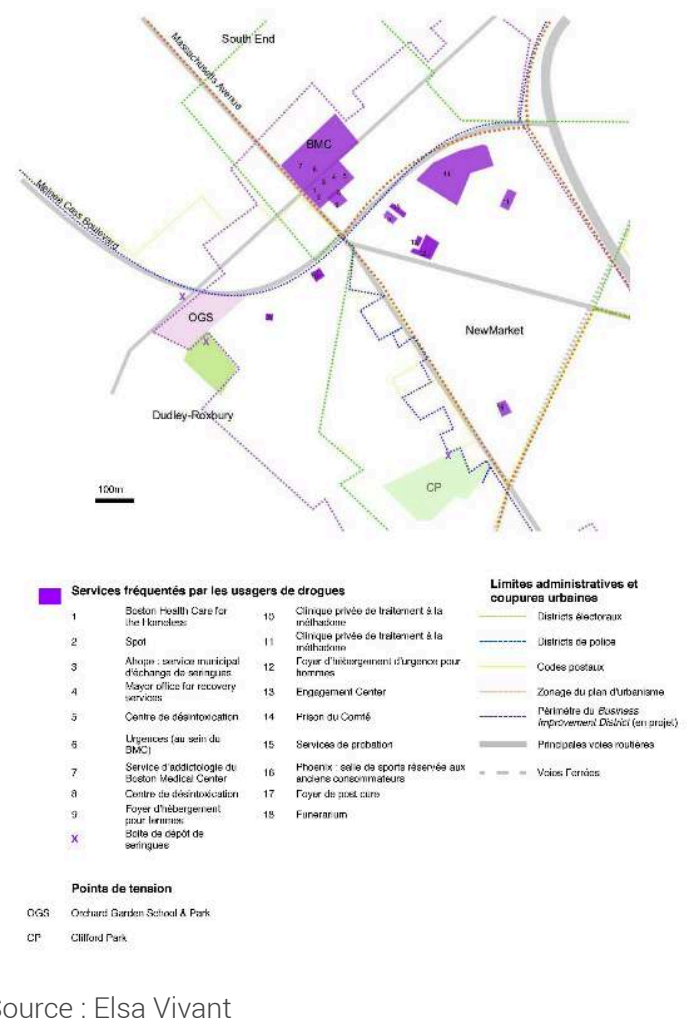

Pour décrire la situation, plusieurs enquêtés font référence à la troisième saison de la série The Wire où, pour répondre à la pression de ses supérieurs demandant une baisse des statistiques de la criminalité, un officier de policier prend l'initiative de tolérer l'usage de drogues dans un périmètre délimité de son secteur d'intervention. Les dealers du quartier sont invités, manu militari, à faire leur commerce uniquement dans une petite rue résidentielle bordée de maisons abandonnées promises à la démolition. Suscitant au départ plus de suspicions que d'enthousiasme, tant du point de vue des agents de police que des dealers, l'expérience semble prometteuse : les corners de vente sont délaissés et apaisés, la criminalité baisse, les consommateurs se pressent dans cette free zone surnommée Hamsterdam ${ }^{8}$. Mais la pseudo-légalisation montre aussi ses limites. En l'absence de contrôle policier, les emplois de guetteurs sont supprimés et les enfants qui les occupaient sont laissés à eux-mêmes; la concentration sur un petit territoire des formes les plus problématiques de consommation les rend plus visibles. Lorsqu'ils font références à cette fiction, les enquêtés expriment leurs craintes d'une normalisation des usages de drogues. Cette préoccupation est particulièrement forte dans les propos de deux enquêtés africains-américains d'une quarantaine d'années, dont le père (et d'autres membres de leur famille) a été consommateur de drogue. Outre le phénomène de légalisation d'une scène de rue, cette saison de la série met en scène un autre processus : celui d'une évolution des pratiques policières, portée par des agents conscients des impasses et limites des politiques qu'ils mettent en œuvre, faisant face à une situation qui les déborde et qu'ils ne contrôlent plus. Or face à une mortalité par surdose qu'ils attribuent à un produit à la dangerosité inégalée et pour laquelle ils n'ont que peu de prise, certains policiers font évoluer leurs pratiques vers 
des approches plus compréhensives dont il conviendrait d'étudier les ressorts et limites (Green et al., 2013).

Illustration 3 - Annonce de bienvenue de la station de lavage automobile située au croisement de Melnea Cass et Mass Av.

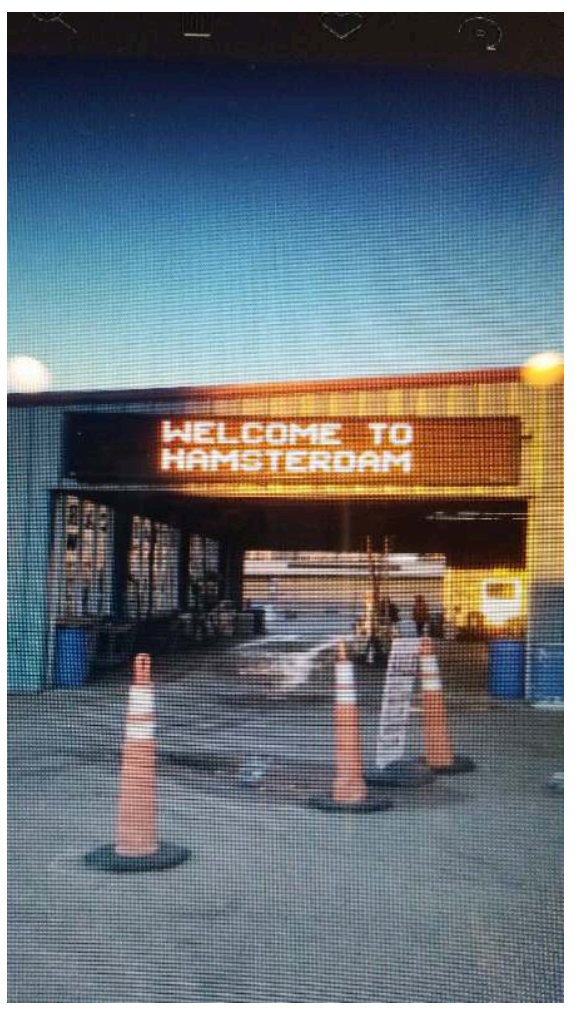

Source : inconnue ${ }^{9}$

13 Plus qu'Amsterdam, l'expérience imaginée dans The Wire rappelle celle que la ville de Zurich a tentée entre 1987 et 1992 pour contenir, géographiquement, les problèmes et désordres liés à l'usage de drogues. Constatant l'inefficacité des pratiques policières de dispersion des usagers, la consommation d'héroïne a été tolérée dans un parc près de la gare centrale (Platzspitz), où ont été expérimentées des pratiques de réduction des risques pour faire face à l'épidémie de Vih (telles que la distribution de seringues) et d'accès aux soins (Grob, 1993 ; Lalande, 2018). Après quelques années, l'expérience a tourné court, la situation sanitaire et sécuritaire étant devenue incontrôlable. Le parc était surnommé Needle Park, stigmatisation toponymique que l'on retrouve dans le nom d'un groupe de riverains de la scène ouverte de Boston sur les réseaux sociaux, A needle in the park, en référence au titre d'un film de Jerry Schatzberg (avec Al Pacino) sorti en 1971 : The Panic in Needle Park. La fermeture du parc zurichois s'est articulée à la mise en place de services permanents de prévention et de médecine pour les usagers de drogues et de dispositifs de réduction des risques, dont les salles de consommation à moindre risque, initiée à Bern dès 1986 (Jauffret-Roustide, 2016), contribuant à l'émergence d'une autre approche du rétablissement. La localisation de ces services dans un espace nommé road plutôt que mile, c'est-à-dire la route plutôt qu'une distance, renforce l'idée que le rétablissement est un processus long et incertain, recovery road faisant écho à l'expression on the road to recovery 


\section{Recovery Road : Requalifier le problème et envisager les solutions}

Pour retourner l'image stigmatisée de Methadone Mile, les médecins et professionnels de la réduction des risques préfèrent utiliser le terme Recovery Road, pour souligne les efforts des différents services d'accompagnement des consommateurs vers la voie du rétablissement ${ }^{10}$, traduction du terme recovery. Notons que ce terme, recovery, est également utilisé pour qualifier les territoires connaissant un regain d'activités et d'attractivité après une période de déprise. Son usage ici est polysémique, le quartier de NewMarket, zone industrielle péricentrale, étant identifié, dans les documents d'urbanisme, comme un territoire à enjeux stratégiques lui-même en recovery. La relocalisation dans le secteur de plusieurs services dédiés au soin en addictologie et/ou à destination des sans-abris a pour conséquences une croissance de la fréquentation des espaces publics par des populations marginalisées. Pour répondre aux demandes des riverains et des entreprises de réduire les nuisances et les risques liés à des comportements jugés déviants et dans une perspective humanitaire de mise à l'abri, la ville a créé, en 2017, un centre d'accueil de jour (appelé Engagement Center) en transformant un chapiteau précédemment utilisé comme espace de stockage. Chauffé l'hiver et climatisé l'été, ce centre est ouvert 12 heures par jour, 7 jours sur 7 , et accueille sans conditions c'est-à-dire sans contrôle d'identité ou de port d'arme et sans obligation de sobriété. Les usagers peuvent se reposer, rencontrer des travailleurs sociaux, accéder à des toilettes, retrouver leurs amis et conjoints ${ }^{11}$, recharger leur téléphone.... Contraints par le site et ses exigences (le lieu n'est qu'une tente, localisé dans une petite rue d'une zone industrielle, entre un foyer d'urgence et une prison, qui ont chacun demandé l'installation de barrière pour éviter les intrusions), ses concepteurs ont cherché dans l'urgence, face à l'hostilité des riverains et avec les moyens du bord, à le rendre le plus accueillant possible par le choix du mobilier, de la peinture du sol pour délimiter les espaces, l'installation de plantes vertes, d'une bibliothèque, d'ordinateurs en accès libre, d'un comptoir de distribution de boissons et snacks. 


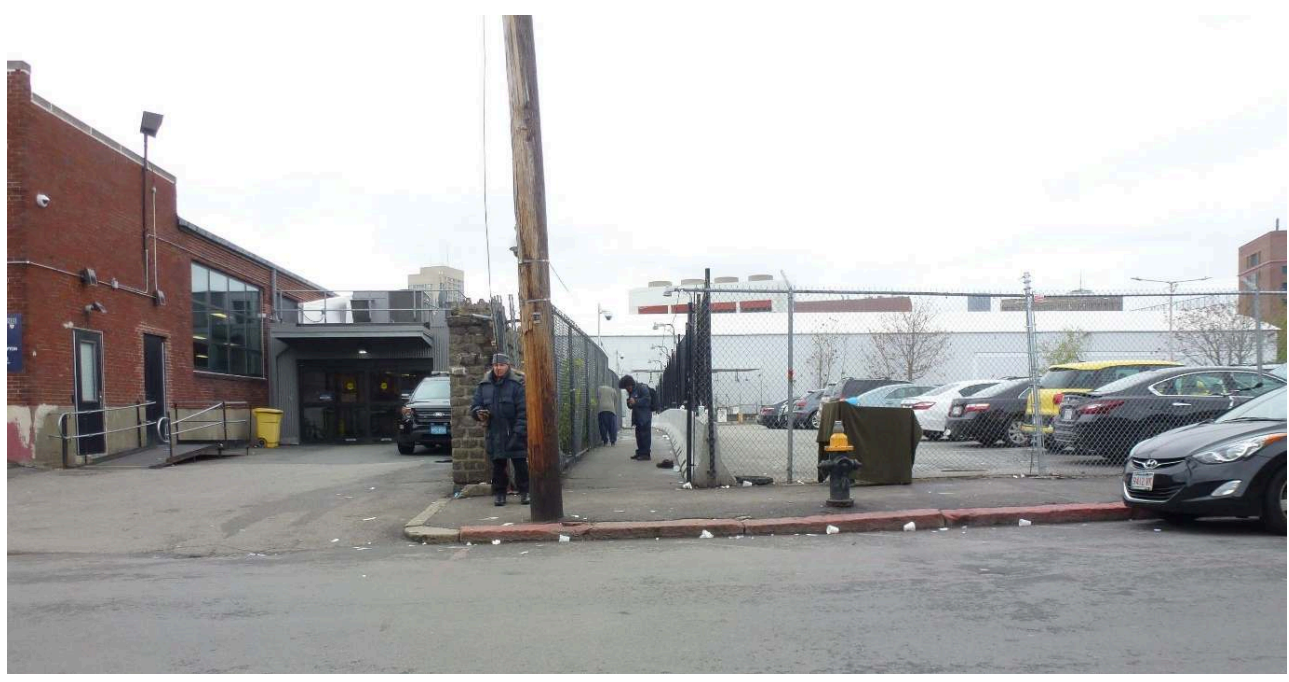

Un corridor de grillage surveillé par trois caméras a été créé pour délimiter l'accès entre l'entrée du centre d'hébergement (à gauche) et le parking des services du shérif et de la prison du comté (à droite). Quelques tables en bois devant l'entrée du chapiteau forment une terrasse entourée de sanitaires mobiles de chantier.

Source : Elsa Vivant.

Ce lieu bénéficie du soutien de l'association d'entreprises qui, malgré ses réticences initiales, juge positivement ses effets sur les usages de l'espace public. Mais il demeure controversé. Certains riverains considèrent qu'il contribue à la cristallisation des conflits d'usage de l'espace public dans le secteur, la vente et la consommation de drogue se déroulant ouvertement devant son entrée, sous le regard de la police, ce qui alimente un second registre de critiques concernant le bon usage de l'argent public ${ }^{12}$. Pensé comme une expérimentation temporaire en réponse à l'urgence, les services de la municipalité envisagent, au moment de l'enquête, sa pérennisation.

Face à la hausse dramatique des surdoses dans le secteur, le centre municipal de soins pour sans-abris a, de son côté, aménagé en 2016 une petite salle de réunion en un espace de repos médicalisé où les usagers peuvent se rendre, juste après avoir consommé, selon le même principe d'accès bas seuil sans conditions que dans l'Engagement center. Dans une lumière tamisée et calme, les usagers sont sous surveillance médicale et des infirmières peuvent intervenir en cas de surdose. Ce service, appelé Spot (Supportive place for observation and treatment), est présenté comme un succès en matière de réduction de la mortalité par surdose, de limitation des usages en espace public, d'accès au soin et d'acceptation par le voisinage. L'Etat du Massachusetts s'en inspire pour définir sa politique de réduction des surdoses.

Pour les acteurs et militants de la réduction des risques, l'Engagement center et Spot ne sont que des pis-aller. Eux défendent l'idée de créer des salles de consommation à moindre risque pour enrayer la recrudescence des infections, réduire le risque de surdose et contribuer à la destigmatisation des usagers en leur destinant un espace adapté à leurs besoins. Existant depuis plusieurs décennies dans certains pays européens ${ }^{13}$, de tels espaces sont pour l'heure interdits aux Etats-Unis. Ces dernières années, dans plusieurs villes américaines, des militants s'organisent pour convaincre les décideurs politiques de modifier la législation afin de rendre possible la création de salle de consommation à moindre risque (Lupick, 2017). A Boston, l'action de la 
coalition Safe Injection Facilities Massachusetts Now! (le terme «Now!» (maintenant!) énonçant l'urgence de la situation) a débuté en 2016 et a rapidement trouvé un écho chez quelques législateurs. Une proposition de loi a été déposée, sans succès, en 2017 ; une commission a été installée en 2018 par le gouverneur et a conclu ses travaux en recommandant l'expérimentation de salles de consommation; le lobbying est en cours pour amener le dépôt d'un nouveau projet de loi lors de la législature 2020. Pour autant, les obstacles sont encore nombreux, et si de plus en plus d'élus sont convaincus de l'intérêt de cette proposition ${ }^{14}$, le gouverneur freine l'avancée législative, le procureur fédéral du Massachusetts menace d'arrestation quiconque travaillerait dans un tel site, et les riverains restent au mieux dubitatifs sur l'effet qu'un tel lieu aurait sur les usages de l'espace public dans leur quartier.

18 Là encore, la question de la nomination est cruciale. Pour les opposants, de tels lieux ne seraient que des shooting galeries (qui véhiculent l'image dégradante d'injection dans de très mauvaises conditions $\mathrm{d}^{\prime}$ hygièn $\mathrm{e}^{15}$ ) ou des heroin den (en référence aux fumeries d'opium). Pour calmer les inquiétudes, certains proposent de les nommer safe consumption space plutôt que safe injection facility (terme initialement choisi par la coalition militante) ce qui permettrait d'occulter l'image stigmatisante de la seringue tout en incluant les consommateurs non injecteurs (notamment les fumeurs de crack). D'autres proposent de les nommer overdose prevention site ou harm reduction site qui n'expriment pas explicitement l'idée d'un usage de drogue mais une finalité de prévention (éviter les surdoses et réduire les risques) qui pourraient se traduire par différentes actions. Il s'agit, par le langage, de renverser le discours dominant par lequel l'addiction est pensée comme une défaillance morale (a moral failure), stigmatisant et culpabilisant l'individu et son entourage (familles, amis, voisinage, communauté), pour présenter les troubles de dépendance comme une maladie. Présenter ces dispositifs comme les premiers jalons vers le rétablissement et point d'entrée dans le parcours de soin est un moyen de convaincre les plus réticents en s'inscrivant dans une logique de gestion du rétablissement (recovery management (Stuart, 2014)) orientant le consommateur dans le droit chemin, celui du soin et peutêtre, un jour, de l'abstinence.

\section{Conclusion : La nomination comme instrument du changement des pratiques et des représentations}

Les différentes nominations de la scène ouverte sont porteuses de plusieurs registres de revendications et viennent rappeler l'importance du choix des mots pour décrire une situation et formuler un problème. Les habitants du quartier pauvre africain-américain de Roxbury dénoncent l'usage de l'adressage (Mass \& Cass) comme toponyme qui minimiserait l'impact de la crise sur leur quartier et occulterait les rapports sociospatiaux de domination. Pour faire face au sentiment d'injustice et d'abandon, ils se mobilisent pour attirer l'attention et l'action de la municipalité sur les atteintes à la qualité de leur cadre de vie, en premier lieu les usages de drogues en espace public et leurs traces que sont les seringues. En insistant sur l'aspect le plus controversé de l'offre de soin avec le nom de Methadone Mile, les riverains de la scène ouverte appellent les autres communautés à prendre en charge leur part du problème. Les professionnels de santé, pour leur part, font valoir les espoirs que de nouvelles pratiques de réduction des risques, pour l'heure à la frontière de la légalité, suscitent en vue de limiter les 
effets (sanitaires, sociaux et létaux) de la consommation. Ils promeuvent ainsi l'appellation Recovery Road qui pourrait annoncer une réhabilitation sociale et urbaine plus globale. Les enjeux politiques de la nomination ne portent pas que sur les lieux. Par les changements de vocabulaire, les militants de la cause des usagers de drogues invitent à changer le regard porté sur la drogue et ses usages. Ainsi, les termes (de plus en plus utilisés) substance use disorder (trouble d'usage de substance) ou people who use drugs (personne qui utilise des drogues) rappellent que de nombreux usagers occasionnels n'ont pas de problème de dépendance. Pour autant, dans les arènes publiques du débat, la voix des principaux concernés, les usagers, reste peu audible bien qu'ils se soient récemment constitués en collectif (Boston Users Union), interviennent sur les réseaux sociaux et ont été invités aux discussions sur la définition des politiques sanitaires.

Enfin, si par la nomination des personnes et des lieux, un léger infléchissement vers des approches plus compassionnelles apparait, les pratiques vexatoires vis-à-vis des sansabris et usagers de drogues demeurent fréquentes. A l'été 2019 , suite à la recrudescence des troubles à l'ordre public, la ville de Boston a mené une opération de police appelée «clean sweep » que l'on peut traduire par «balayage sans ménagement ", qui a donné lieu à la destruction des biens des sans-abris et à plusieurs arrestations. Ce vocabulaire et ces procédés rappellent les heures les plus sombres de la guerre à la drogue et les logiques de bannissement qui gouvernent la vie des sans-abris (Beckett and Herbert, 2009). Par la suite, la municipalité a déployé un plan d'action pour améliorer la qualité de vie, dont le nom Mass \& Cass 2.0 reprend l'adressage et, dans une référence au web 2.0, exprime l'idée d'un renouveau plus participatif dans la définition des politiques de gestion de l'espace public (la question étant de savoir qui du riverain ou de l'usager de drogue est invité à participer). Pourtant, les demandes des riverains ne se limitent pas à une demande d'intervention policière; ils envisagent également des évolutions dans l'offre de soin, sa distribution (éclatée plutôt que concentrée) et ses modalités thérapeutiques. Ils soutiennent aussi le projet de la Ville de Boston de reconstruire le pont de Long Island pour réinstaller des services de soin en addictologie sur l'ile. Dit autrement, de (re-)déplacer le problème loin des regards.

\section{BIBLIOGRAPHIE}

Ahrnsbrak R., Bose J., Hedden S., et al., 2017. Key Substance Use and Mental Health Indicators in the United States: Results from the 2016 National Survey on Drug Use and Health, No. SMA 17-5044, NSDUH Series H-52. Rockville, MD: Substance Abuse and Mental Health Services Administration.

Alexander M., 2017. La couleur de la justice. Incarcération de masse et nouvelle ségrégation raciale aux Etats-Unis. Editions Syllepse, Paris. 
Bearnot B., Rodriguez J., Pearson J., 2018. Crowdsourcing the Opioid Epidemic. Public Health Post, 6 septembre 2018. https://www.publichealthpost.org/viewpoints/crowdsourcing-the-opioidepidemic/

Beckett K., Herbert S., 2009. Banished. The New Social Control in Urban America. Oxford University Press, Oxford.

City of Boston, 2017. Imagine Boston 2030. City of Boston, Boston.

Clark C., 2017. The Recovery Revolution. The Battle Over Addiction Treatement in the United States. Columbia University Press, New York.

Fitzgerald B., 2017. Supervised Injection Facilities: Harm or Harm Reduction? Public Health Post, 16 juillet 2017. https://www.publichealthpost.org/essays/we-can-do-better/

Giraut F., Houssay-Holzschuch M., Guyot, S., 2008. Au nom des territoires : Enjeux géographiques de la toponymie. L'Espace géographique, vol. 37, n² 2, p. 97-105.

Green T., Zaller N., Palacios W., et al., 2013. Law enforcement attitudes toward overdose prevention and response. Drug and Alcohol Dependence, $\mathrm{n}^{\circ}$ 113, p. 677-684.

Greenberg Z., 2019. 'A new day': Dudley Square is renamed Nubian Square. Boston Globe, 19 décembre 2019.

Grob P.-J., 1993. The needle park in Zürich. The story and the lessons to be learned. European Journal on Criminal Policy and Research, vol. 1, n 2, p. 48-60.

Jauffret-Roustide M., 2016. Les salles de consommation à moindre risque. Apprendre à vivre avec les drogues. Esprit, $\mathrm{n}^{\circ} 11$, p. 115-123.

King M., 1981. Chain of Change. Struggles For Black Community Development. South End Press, Boston. Lupick T., 2017. Fighting for Space. How a group of drug users transformed one city's struggle with addiction. Arsenal Pulp Press. Vancouver.

MacQuarrie B., 2019. Dudley Square: at the intersection of Colonial history, African heritage. Boston Globe. 18 décembre2019.

Massachusetts Department of Public Health, 2016. Chapter 55. An assessment of Opioid-Related Deaths in Massachusetts (2013-14). Boston, The Commonwealth of Massachusetts.

Meier B., 2003. Pain Killer. A 'Wonder' Drug's Trail of Addiction and Death. Rodale, New York.

Meyers T., 2016. La clinique et ailleurs. Anthropologie et thérapeutique de l'addiction. Editions Vrin, Paris.

Miller M. B. ed., 2018. Boston's Banner Years: 1965-2015. A Saga of Black Success. Archway Publishing, Bloomington, In.

Musto D. F., 1999 (3rd edition). The American Disease. Origins of Narcotic Control. Oxford University Press, New York.

Netherland J., Hansen H., 2016. The War on Drugs that Wasn't: Wasted Whiteness, 'Dirty Doctors', and Race in Media Coverage of Prescription Opioid Misuse. Culture, Medecine and Psychiatry, vol. 40, n 4, p. 664-686.

O'Connor T., 2001. The Hub. Boston Past and Present. Northeastern University Press, Boston.

Platt J., Widman M., Lidz V., Marlowe D., 1998. Methadone Maintenance Treatment: Its Development and Effectiveness after 30 years. In Inciardi J., Harrison L.(ed.), Heroin in the Age of Crack-Cocaine. Sage, Thousand Oaks. 
Rose-Redwood R., Alderman D., 2011. Critical Interventions in Political Toponymy. ACME: An International E Journal for Critical Geographies [En ligne], vol.10, n 1, p. 1-6. URL: https://acmejournal.org/index.php/acme/article/view/879

Schneider E. C., 2011. Smack. Heroin and the American City. University of Pennsylvania Press. Philadelphie.

Stuart F., 2014. From 'Rabble Management' to 'Recovery Management': Policing Homelessness in Marginal Urban Space. Urban Studies, vol. 51, n 9, p. 1909-1925.

Tissot S., 2011. De bons voisins. Enquête dans un quartier de la bourgeoisie progressiste. Raisons d'agir, Paris.

White W. L., 1998. Slaying the Dragon. The History of Addiction Treatment and Recovery in America. Chestnut Health System, Normal, Illinois.

\section{NOTES}

1. Qu'ils soient légaux (médicaments antalgiques) ou illégaux (héroïne), obtenu par des voies légales (sur prescription) ou sur le marché clandestin, le mésusage d'opioïdes concernerait près de douze millions de personnes aux Etats-Unis, dont deux millions présenteraient un trouble addictif (Ahrnsbrak et al., 2017).

2. Au Massachusetts, le nombre de décès par surdose a plus que doublé entre 2013 et 2016 pour atteindre plus de 2000 décès par an (Massachusetts Department of Public Health, 2016).

3. Auprès de professionnels des forces de l'ordre (police, justice), de santé et de la réduction des risques, d'élus locaux, de riverains, de parents de toxicomanes et de militants pour les droits des usagers.

4. Réunions de parents d'élèves, d'associations de riverains, de groupes militants pour les droits des usagers de drogues, de groupe de parole de parents de toxicomanes, du conseil municipal, de la commission de l'Etat pour la réduction des risques et réunions publiques de quartier.

5. La gentrification du South End se poursuit vers Dudley/Nubian Square alors que les inégalités de richesses entre les ménages blancs et les ménages africain-américains sont incommensurables : un rapport officiel établit que le patrimoine moyen des ménages blancs est de 247000 \$ alors que celui des ménages africains-américains est de 8\$ (City of Boston, 2017).

6. Un rapide sondage dans la presse locale (Boston Globe et Boston Herald) via la plateforme Factiva a dénombré 111 articles publiés depuis 2015 comprenant l'expression Methadone Mile et aucun utilisant le terme Recovery Mile privilégié par les activistes (cf. supra).

7. Au moment de l'enquête, ce projet est en discussion.

8. L'ajout d'un « H » produit un jeu de mots entre Ham (le jambon) qui renvoie à Pig (cochon), le surnom argotique des policiers, et Amsterdam.

9. Cette photographie m'a été transmise par une enquêtée, professionnelle du soin en addictologie intervenant dans le quartier. Il est fait allusion à cette enseigne dans un billet de blog (Fitzgerald, 2017).

10. Les attentes en termes de guérison et de rétablissement ne sont pas les mêmes selon que l'on est consommateur, médecin ou proche (Meyers, 2016). Cela renvoie également au débat au sein du monde médical quant aux modalités d'accompagnement et de prise en charge des personnes souffrant de troubles addictifs, entre les tenants de l'abstinence et les partisans de la réduction des risques. Les centres de soin visant l'abstinence comme moyen et finalité du traitement sont le plus souvent à l'écart des communautés. Au contraire, les approches en termes de réduction des 
risques prennent en charge la personne là où elle est, le là où renvoyant à la fois au territoire de vie de la personne et à sa trajectoire de consommation et aux objectifs de soin qu'elle se fixe.

11. Aucun centre d'hébergement de la ville ne propose d'accueil pour les couples.

12. Le dispositif coûte environ 2 millions de dollars à la Ville de Boston (entre les coûts d'aménagement et de fonctionnement, notamment par l'emploi de huit travailleurs sociaux dédiés).

13. En France, les premières salles de consommation à moindre risque ont été créées en 2016 dans le cadre de dispositifs expérimentaux.

14. En premier lieu le maire de Boston qui met publiquement en scène son engagement personnel sur le sujet en raison de son passé d'alcoolique, et l'évolution de son point de vue sur les salles de consommation suite à la visite de plusieurs sites au Canada.

15. Si dans ce contexte shooting galery se traduit en français salle de shoot, ce terme désigne aussi un stand de tir.

\section{RÉSUMÉS}

Cet article analyse les différentes toponymies d'une scène ouverte de consommation de drogues, à Boston, localisée à l'interface de trois quartiers aux usages et dynamiques contrastées. Les réactions et demandes des riverains de ces différents quartiers sont révélateurs des rapports de pouvoir dans l'espace urbain dont les choix toponymiques sont les analyseurs: Mass \& Cass renvoie à l'histoire urbaine, coloniale et raciale de la ville et de la prohibition; Methadone Mile rappelle la stigmatisation des usagers et des lieux de soin ; Recovery Road exprime l'émergence de nouvelles pratiques de soin et de réduction des risques à l'œuvre dans ce secteur. Entrer sur cette scène par ses noms permet de dénouer la complexité de la crise des opioïdes en cours aux USA, de ses enjeux politiques et des infléchissements qu'elle provoque dans la politique des drogues.

This paper analyses the different toponyms of an open drug use scene in Boston, located at the interface of three neighborhoods with contrasting uses and dynamics. The reactions and demands of the residents of these different neighbourhoods reveal the power relationships in the urban space, the toponymic choices of which are the main focus of the analysis: Mass \& Cass refers to the urban, colonial and racial history of the city and prohibition; Methadone Mile recalls the stigmatisation of users and places of care; Recovery Road expresses the emergence of new care and harm reduction practices at work in this sector. Entering this scene by name helps to unravel the complexity of the opioid crisis currently underway in the USA, the political stakes involved and the changes it is driving in drug policy.

\section{INDEX}

Mots-clés : Boston, opioïdes, surdose, scène ouverte, dynamiques socio-spatiales

Keywords : Boston, opioid, overdose, open drug scene, socio-spatial dynamics

Thèmes : Sur le Champ - Sur le Terrain 


\section{AUTEUR}

\section{ELSA VIVANT}

Elsa Vivant, elsa.vivant@univ-paris-est.fr, est maître de conférences à l'Université Paris Est Marne-la-Vallée. Elle a récemment publié :

- Vivant E., 2020. From margins to capital: The integration of spaces of artistic critique within capitalist urbanism. Journal of Urban Affairs, $5^{\text {th }}$ octobre, p. 1-14.

- Vivant E., 2018. Accompagner l'implantation d'un établissement de soins psychiatriques. Les enseignements d'un atelier de programmation urbaine avec les usagers. Cahier Ramau, $\mathrm{n}^{\circ} 9$, p. 86-101.

- Vivant E., 2018. Les fantômes de la Tour Utrillo: métonymie de l'action urbaine à Clichy-sousbois et Montfermeil. Géographie et culture, n 106, p. 25-42. 